\title{
Systemic and Renal Hemodynamics in Oliguric Hepatic Failure: Effect of Volume Expansion*
}

\author{
Felix E. Tristani and Jay N. CoH N \\ (From the Medical Service, Veterans Administration Hospital and the Department of Medicine, \\ Georgetozen University Medical Center, Washington, D. C.)
}

\begin{abstract}
Systemic and renal hemodynamics were studied by indicator dilution techniques before and after infusion of $500 \mathrm{ml}$ of dextran 40 in 21 patients with renal failure developing in the course of decompensated cirrhosis.

Cardiac index was directly correlated with total blood volumes. Renal blood flow was low and renal vascular resistance elevated in 13 of 15 patients. Renal vascular resistance was directly related to total systemic nonrenal vascular resistance. Two patients with the highest cardiac outputs in the group were oliguric with high renal blood flow.

Plasma volume expansion increased cardiac output in 19 of 21 patients and increased renal blood flow in 12 of 14 . The patients were divided into two groups on the basis of control cardiac index. Those with lower cardiac index had lower blood volumes and responded to dextran with a $73 \%$ increase in cardiac output, a $148 \%$ increase in renal blood flow, and a rise in renal fraction. Those with high control cardiac index had significantly higher blood volumes and responded to dextran with only a small average rise in cardiac output and renal blood flow.

Systolic arterial pressure was less than $100 \mathrm{~mm} \mathrm{Hg}$ in 12 patients. When compared to the normotensive subjects, this hypotension was characterized by a lower vascular resistance, a tendency for a lesser rise in renal blood flow after volume expansion, and a more rapid demise.

The prompt circulatory improvement after volume expansion in many of these patients indicates that functional plasma volume depletion may be an important factor in the renal vasoconstriction of oliguric hepatic failure. In an attempt to sustain volume expansion, reinfusion of ascitic fluid was accomplished in four patients. Normal renal blood flow was maintained during reinfusion and a diuresis always occurred, but the response usually was not maintained after the infusion was terminated.
\end{abstract}

\section{Introduction}

Oliguria and azotemia may develop spontaneously in patients with severe liver disease, and the syndrome usually carries a grave prognosis. De-

* Received for publication 17 March 1967 and in revised form 24 July 1967.

This study was supported in part by U. S. Public Health Service Research Grant HE-0978501 from the National Heart Institute and a Grant-in-Aid from the Washington Heart Association.

Address requests for reprints to Dr. Jay N. Cohn, V. A. Hospital, 50 Irving Street, N.W., Washington, D. C. 20422. spite numerous clinical studies the mechanism of the renal failure in this setting has remained conjectural $(1,2)$.

The absence of a history of kidney disease or of a specific histological renal lesion has led to the suggestion that the renal failure may be due to decreased renal perfusion ( 3 ), and clearance studies have suggested that renal blood flow is severely reduced $(4,5)$. However, the mechanism of the renal hemodynamic abnormality in this syndrome has not been established. Since depletion of intravascular volume may lead to renal vasocon- 
TABLE I

Clinical data

\begin{tabular}{|c|c|c|c|c|c|c|c|c|}
\hline Patient & Age & $\begin{array}{c}\text { Blood urea } \\
\text { nitrogen }\end{array}$ & $\underset{\text { bilirubin }}{\text { Serum }}$ & $\underset{\mathrm{Na}}{\text { Serum }}$ & $\underset{\mathbf{K}}{\text { Serum }}$ & $\frac{\text { Albumin }}{\text { Globulin }}$ & Hematocrit & $\underset{\text { time }}{\text { Survival }}$ \\
\hline & $y r$ & $\mathrm{mg} / 100 \mathrm{ml}$ & $\mathrm{mg} / 100 \mathrm{ml}$ & $m E q / l i t e r$ & $m E q /$ liter & $\mathrm{mg} / 100 \mathrm{ml}$ & $\%$ & days \\
\hline 1 & 46 & 62 & 2.4 & 125 & 3.7 & $2.2 / 4.0$ & 31 & 54 \\
\hline 2 & 59 & 62 & 7.7 & 113 & 5.3 & $3.9 / 3.3$ & 40.7 & 11 \\
\hline 3 & 62 & 27 & 3.7 & 130 & 3.6 & $1.4 / 3.9$ & 28.5 & 16 \\
\hline 4 & 47 & 63 & 7.7 & 123 & 2.8 & $3.0 / 3.0$ & 37 & 37 \\
\hline 5 & 47 & 60 & 16.0 & 129 & 4.3 & $1.5 / 1.9$ & 24 & 5 \\
\hline 6 & 40 & 78 & 24.6 & 121 & 4.6 & $2.2 / 3.3$ & 39.5 & 7 \\
\hline 7 & 57 & 67 & 20.4 & 129 & 3.5 & - & 36 & 7 \\
\hline 8 & 48 & 142 & 17.8 & 127 & 4.1 & $2.2 / 2.9$ & 30 & 5 \\
\hline 9 & 37 & 32 & 3.9 & 123 & 2.1 & $2.9 / 2.7$ & 32 & 16 \\
\hline 10 & 69 & 80 & 15.5 & 127 & 2.7 & $2.2 / 5.2$ & 53 & 1 \\
\hline 11 & 57 & 118 & 2.9 & 142 & 3.2 & $2.3 / 3.3$ & 26 & 12 \\
\hline 12 & 59 & 29 & 1.8 & 131 & 4.3 & $2.9 / 3.6$ & 43 & 21 \\
\hline 13 & 54 & 32 & 1.8 & 131 & 3.4 & $3.1 / 3.2$ & 35 & 3 \\
\hline 14 & 37 & 36 & 3.4 & 130 & 3.0 & $1.7 / 4.0$ & 27.6 & 4 \\
\hline 15 & 46 & 12 & 4.3 & 126 & 3.6 & $3.4 / 2.2$ & 28 & 13 \\
\hline 16 & 47 & 19 & 8.4 & 125 & 4.6 & $2.0 / 4.0$ & 27.5 & 24 \\
\hline 17 & 37 & 41 & 19.2 & 138 & 2.8 & $2.4 / 1.8$ & 40.0 & 3 \\
\hline 18 & 57 & 150 & 31.0 & 116 & 3.4 & $1.9 / 3.4$ & 32.5 & 6 \\
\hline 19 & 46 & 30 & 13.0 & 118 & 3.4 & $2.0 / 2.2$ & 33 & alive \\
\hline 20 & 55 & 63 & 14.5 & 133 & 4.7 & $2.2 / 3.1$ & 33 & 4 \\
\hline 21 & 46 & 50 & 35.0 & 124 & 2.4 & $2.7 / 5.0$ & 27 & 12 \\
\hline
\end{tabular}

striction (6) and the cirrhotic may pool large quantities of extracellular fluid in areas not available to the circulation, it has been suggested by Vesin (7) and Papper, Belsky, and Bleifer (3) that an effective volume deficit is an important factor contributing to the renal failure.

If reduced circulating volume is the cause of renal hypoperfusion, then volume expansion should tend to correct it. The purpose of the present study was to evaluate systemic and renal hemodynamics in a group of cirrhotic patients who developed oliguric renal failure and to measure the circulatory response to acute plasma volume expansion.

\section{Methods}

\section{Patients studied}

21 male patients (age range from 37 to $69 \mathrm{yr}$ ) on the medical wards of the Veterans Administration Hospital with the diagnosis of cirrhosis of the liver associated with oliguria (urine output less than $350 \mathrm{cc}$ in $24 \mathrm{hr}$ ) were studied. The diagnosis of liver cirrhosis was based on a history of alcoholism, hepatomegaly, jaundice, and the presence of stigmata of the disease, such as angiomata, gynecomastia, testicular atrophy, and palmar erythema. All had moderate to marked ascites, and encephalopathy had been present at some time during the hospital course in every patient. The blood urea nitrogen on admission averaged $17 \mathrm{mg} / 100 \mathrm{ml}$ and had increased to 59.7 $\mathrm{mg} / 100 \mathrm{ml}$ by the day of study. Results of laboratory studies in these patients are shown in Table $I$.
Tissue diagnosis of cirrhosis of the liver was established in all 16 patients upon postmortem examination and in an additional 2 by liver biopsy. All examined kidneys showed bile staining. In two subjects focal thickening of the glomerular basement membrane was demonstrated. No other specific renal abnormalities were found on histological examination by light microscopy. One patient had a hepatoma and in two others incidental carcinoma of the esophagus was found at autopsy.

\section{Procedures}

Studies were performed at the patients' bedside or in the hemodynamics laboratory. In five patients a Cournand needle was introduced percutaneously into the femoral artery and a vinyl catheter was threaded into the right atrium through a $14 \mathrm{G}$ thin-walled needle placed in the femoral vein. In the other 16 patients, radiopaque polyethylene catheters were introduced into a femoral artery and vein using a Seldinger technic. The catheters were advanced into the renal artery and corresponding renal vein under direct visualization with a portable image intensifier. The position in the renal vessels was confirmed in some instances by the injection of $1-2 \mathrm{cc}$ of $50 \%$ Hypaque.

Arterial and venous pressures were recorded with Statham P23Db strain gage transducers, carrier preamplifiers, and a multichannel direct writing Sanborn recorder, using the mid-chest as the zero reference level. Mean pressures were obtained by electronic integration. Renal blood flow ( $R B F$ ) was measured in individual kidneys by an indicator dilution technic (8). $\frac{1}{2}$ $\mathrm{ml}(0.6 .25 \mathrm{mg})$ of indocyanine green dye (Cardiogreen) was injected into the dye-filled catheter in the renal artery while renal venous blood was drawn at a constant 
rate through a Gilford cuvette densitometer using a Gilford constant flow pump. Cardiac output (CO) also was determined by the indicator-dilution method, with injection of $5 \mathrm{mg}$ of indocyanine green dye into the right atrium or renal vein while sampling from the aorta, femoral artery, or renal artery through the same cuvette. In order to follow hemodynamic response during the study, a Lexington cardiac output computer was used to give an immediate read-out of $\mathrm{CO}$ and $\mathrm{RBF}$. All dye curve flows also were calculated manually by the standard Stewart-Hamilton method (9). Values reported for CO represent the average of two successive determinations; those for $\mathrm{RBF}$, the average of two or three successive curves that varied by less than $10 \%$. Renal blood volume (RBV) in milliliters per kidney was calculated as the product of $\mathrm{RBF}$ and the mean dye transit time, which was corrected for catheter and cuvette delay. Total systemic vascular resistance (SVR) and renal resistance (RR) expressed in dynes-second- $\mathrm{cm}^{-5}$ were calculated from the formulas:

$$
\mathrm{SVR}=\frac{(\text { MAP }-\mathrm{RAP}) \times 1332 \times 60}{\mathrm{CO}}
$$

and

$$
R R=\frac{(\text { MAP }- \text { RVP }) \times 1332 \times 60}{R B F \times 2}
$$

where MAP is mean arterial pressure in $\mathrm{mm} \mathrm{Hg}$, RAP is mean right atrial pressure in $\mathrm{mm} \mathrm{Hg}, \mathrm{RVP}$ is renal venous pressure in $\mathrm{mm} \mathrm{Hg}, \mathrm{CO}$ is cardiac output in milliliters per minute and $\mathrm{RBF}$ is renal blood flow in milliliters per minute per kidney. $\mathrm{RBF}$ is multiplied by two to calculate total $R R$ assuming equal flow to both kidneys. The renal fraction ( $R F$ ) was taken as twice the RBF multiplied by 100 and divided by the $C O$.

Blood volume was measured using a calibrated injection of T-1824. Four arterial blood samples were collected from 10 to $40 \mathrm{~min}$ after injection of the dye and the slope of disappearance was extrapolated semilogarithmically to the time of injection for measurement of plasma volume. The effect of dextran on plasma volume was determined in some subjects by collecting blood samples at intervals before and after dextran infusion. The difference between the T-1824 concentration after dextran and the predicted T-1824 concentration, determined from the disappearance slope before dextran, was used to calculate the expansion of plasma volume that had occurred. Total blood volume was calculated from the plasma volume with the arterial hematocrit corrected for plasma trapping (10) and for the discrepancy betwen large vessel and total body hematocrit (11).

After control studies the patients were given a rapid intravenous infusion of $500 \mathrm{ml}$ of low molecular weight dextran (dextran 40; Rheomacrodex ${ }^{1}$ ) over a 15-20 min period. Hemodynamic studies were repeated immediately after the infusion was completed. In one patient RBF was measured in the control period but was not reassessed after dextran because the catheters could not be repositioned in the renal vessels.

1 Kindly supplied by Pharmacia Laboratories, Inc., New Market, N. J.
Statistical analyses were carried out by standard techniques with rank analysis (12).

\section{Results}

\section{Systemic hemodynamics (Table II)}

During the control period in these 21 patients, arterial pressure averaged $98 / 55 \mathrm{~mm} \mathrm{Hg}$ with a mean of $69.9 \mathrm{~mm} \mathrm{Hg}$. Cardiac index (CI) averaged 3.64 liters/min per $\mathrm{m}^{2}$ and the calculated total systemic vascular resistance (SVR) was 1028 dynes-sec- $\mathrm{cm}^{-5}$. The mean right atrial pressure (RAP) averaged $1.9 \mathrm{~mm} \mathrm{Hg}$. A significant correlation was noted between CI and blood volume ( $r=0.507, P<0.05)$.

The patients were divided into two groups on the basis of control CI. 13 patients with a low or normal CI of less than 3.7 liters/min (Group I) were separated from the other eight patients who exhibited a distinctly elevated CI of over 3.9 liters/ min (Group II). CI averaged 2.53 liters $/ \mathrm{min}$ in Group I and 5.45 liters/min in Group II. Arterial pressure was not significantly different in the two groups, averaging $100 / 56 \mathrm{~mm} \mathrm{Hg}$ (mean $69.1 \mathrm{~mm} \mathrm{Hg}$ ) in Group I and 94/53 $\mathrm{mm} \mathrm{Hg}$ (mean $71.4 \mathrm{~mm} \mathrm{Hg}$ ) in Group II. SVR was therefore considerably higher in Group I, averaging 1295 dynes-sec-cm ${ }^{-5}$ compared to 594 dynes$\mathrm{sec}-\mathrm{cm}^{-5}$ in Group II. Since heart rate was similar in the two groups, stroke volume was much higher in Group II, averaging $106.7 \mathrm{ml}$ compared to $48.2 \mathrm{ml}$ in Group I. Blood volume measured in 19 of the patients averaged $58.3 \mathrm{ml} / \mathrm{kg}$ in Group I and $77.7 \mathrm{ml} / \mathrm{kg}$ in Group II $(P<0.025)$. Four of the seven patients in Group II, but none of the 12 patients in Group I, had blood volumes greater than $70 \mathrm{ml} / \mathrm{kg}$.

The patients also could be divided on the basis of control arterial pressure. Systolic arterial pressure was less than $100 \mathrm{~mm} \mathrm{Hg}$ at the time of study in 12 of the 21 subjects. SVR was lower in these hypotensive patients, averaging 943 dynes-sec- $\mathrm{cm}^{-5}$ compared to 1142 dynes-sec- $\mathrm{cm}^{-5}$ in the normotensive group, but no other hemodynamic differences could be demonstrated.

\section{Renal hemodynamics (Table III)}

Renal dye curves were recorded in 16 of the patients. In one patient, No. 11, the curve was extremely prolonged and of such low amplitude 
TABLE II

Systemic hemodynamics

\begin{tabular}{|c|c|c|c|c|c|c|c|c|c|c|c|}
\hline Patient & BSA & Period & $\begin{array}{l}\text { Arterial } \\
\text { pressure }\end{array}$ & $\begin{array}{c}\text { Mean } \\
\text { arterial } \\
\text { pressure }\end{array}$ & $\begin{array}{l}\text { Cardiac } \\
\text { output }\end{array}$ & $\underset{\text { index }}{\text { Cardiac }}$ & $\begin{array}{l}\text { Systemic } \\
\text { vascular } \\
\text { resistance }\end{array}$ & $\begin{array}{c}\text { Right } \\
\text { atrial } \\
\text { pressure }\end{array}$ & $\begin{array}{l}\text { Heart } \\
\text { rate }\end{array}$ & $\begin{array}{l}\text { Stroke } \\
\text { volume }\end{array}$ & $\begin{array}{c}\text { Blood } \\
\text { volume }\end{array}$ \\
\hline & $m^{2}$ & & $m m \mathrm{Hg}$ & $m m \mathrm{Hg}$ & liters/min & $\begin{array}{l}\text { liters } / \\
\text { min per } \\
m^{2}\end{array}$ & $\begin{array}{c}\text { dymes- } \\
\text { sec-cm-s }\end{array}$ & $m m \mathrm{Hg}$ & per min & $m l$ & $m l / k g$ \\
\hline 1 & 1.96 & $\begin{array}{l}\text { Control } \\
\text { Dextran }\end{array}$ & $\begin{array}{l}120 / 72 \\
106 / 64\end{array}$ & $\begin{array}{l}84 \\
80\end{array}$ & $\begin{array}{l}3.60 \\
8.26\end{array}$ & $\begin{array}{l}1.84 \\
4.21\end{array}$ & $\begin{array}{r}1,800 \\
717\end{array}$ & $\begin{array}{l}3 \\
6\end{array}$ & $\begin{array}{r}98 \\
108\end{array}$ & $\begin{array}{l}37.0 \\
76.0\end{array}$ & 56 \\
\hline 2 & 1.96 & $\begin{array}{l}\text { Control } \\
\text { Dextran }\end{array}$ & $\begin{array}{l}132 / 74 \\
156 / 78\end{array}$ & $\begin{array}{r}92 \\
102\end{array}$ & $\begin{array}{l}4.65 \\
7.46\end{array}$ & $\begin{array}{l}2.37 \\
3.81\end{array}$ & $\begin{array}{l}1,548 \\
1,008\end{array}$ & $\begin{array}{l}2 \\
8\end{array}$ & $\begin{array}{r}102 \\
96\end{array}$ & $\begin{array}{l}45.6 \\
77.7\end{array}$ & 51 \\
\hline 3 & 1.53 & $\begin{array}{l}\text { Control } \\
\text { Dextran }\end{array}$ & $\begin{array}{l}121 / 72 \\
120 / 80\end{array}$ & $\begin{array}{l}88 \\
94\end{array}$ & $\begin{array}{l}4.76 \\
8.04\end{array}$ & $\begin{array}{l}3.11 \\
5.25\end{array}$ & $\begin{array}{r}1,462 \\
826\end{array}$ & $\begin{array}{r}1 \\
11\end{array}$ & $\begin{array}{l}120 \\
120\end{array}$ & $\begin{array}{l}39.7 \\
67.0\end{array}$ & 63 \\
\hline 4 & 1.70 & $\begin{array}{l}\text { Control } \\
\text { Dextran }\end{array}$ & $\begin{array}{r}98 / 48 \\
112 / 52\end{array}$ & $\begin{array}{l}62 \\
70\end{array}$ & $\begin{array}{l}4.42 \\
6.29\end{array}$ & $\begin{array}{l}2.60 \\
3.70\end{array}$ & $\begin{array}{r}1,100 \\
824\end{array}$ & $\begin{array}{l}1 \\
6\end{array}$ & $\begin{array}{r}102 \\
96\end{array}$ & $\begin{array}{l}43.4 \\
65.5\end{array}$ & 48 \\
\hline 5 & 1.79 & $\begin{array}{l}\text { Control } \\
\text { Dextran }\end{array}$ & $\begin{array}{r}84 / 40 \\
100 / 40\end{array}$ & $\begin{array}{l}56 \\
58\end{array}$ & $\begin{array}{l}6.49 \\
9.77\end{array}$ & $\begin{array}{l}3.62 \\
5.46\end{array}$ & $\begin{array}{l}690 \\
434\end{array}$ & $\begin{array}{l}0 \\
5\end{array}$ & $\begin{array}{l}80 \\
78\end{array}$ & $\begin{array}{r}81.2 \\
125.2\end{array}$ & 55 \\
\hline 6 & 1.54 & $\begin{array}{l}\text { Control } \\
\text { Dextran }\end{array}$ & $\begin{array}{r}86 / 62 \\
116 / 68\end{array}$ & $\begin{array}{l}66 \\
84\end{array}$ & $\begin{array}{r}5.49 \\
10.82\end{array}$ & $\begin{array}{l}3.57 \\
7.03\end{array}$ & $\begin{array}{l}949 \\
534\end{array}$ & $\begin{array}{l}1 \\
8\end{array}$ & $\begin{array}{l}120 \\
112\end{array}$ & $\begin{array}{l}45.8 \\
96.6\end{array}$ & 57 \\
\hline 7 & 1.85 & $\begin{array}{l}\text { Control } \\
\text { Dextran }\end{array}$ & $\begin{array}{l}70 / 44 \\
88 / 48\end{array}$ & $\begin{array}{l}52 \\
62\end{array}$ & $\begin{array}{l}3.51 \\
9.68\end{array}$ & $\begin{array}{l}1.90 \\
5.23\end{array}$ & $\begin{array}{r}1,180 \\
479\end{array}$ & $\begin{array}{l}0.5 \\
4\end{array}$ & $\begin{array}{l}111 \\
102\end{array}$ & $\begin{array}{l}31.6 \\
95.0\end{array}$ & 57 \\
\hline 8 & 1.75 & $\begin{array}{l}\text { Control } \\
\text { Dextran }\end{array}$ & $\begin{array}{l}156 / 76 \\
148 / 68\end{array}$ & $\begin{array}{l}96 \\
92\end{array}$ & $\begin{array}{l}5.66 \\
7.22\end{array}$ & $\begin{array}{l}3.23 \\
4.13\end{array}$ & $\begin{array}{r}1,345 \\
898\end{array}$ & $\begin{array}{l}1 \\
9\end{array}$ & $\begin{array}{l}96 \\
90\end{array}$ & $\begin{array}{l}59.0 \\
72.9\end{array}$ & 68 \\
\hline 9 & 1.43 & $\begin{array}{l}\text { Control } \\
\text { Dextran }\end{array}$ & $\begin{array}{l}80 / 40 \\
92 / 42\end{array}$ & $\begin{array}{l}46 \\
56\end{array}$ & $\begin{array}{l}3.18 \\
5.74\end{array}$ & $\begin{array}{l}2.23 \\
4.02\end{array}$ & $\begin{array}{r}1,260 \\
752\end{array}$ & $\begin{array}{r}-4 \\
2\end{array}$ & $\begin{array}{l}108 \\
108\end{array}$ & $\begin{array}{l}29.4 \\
56.3\end{array}$ & 64 \\
\hline 10 & 1.70 & $\begin{array}{l}\text { Control } \\
\text { Dextran }\end{array}$ & $\begin{array}{r}84 / 48 \\
120 / 68\end{array}$ & $\begin{array}{l}60 \\
86\end{array}$ & $\begin{array}{l}2.31 \\
5.63\end{array}$ & $\begin{array}{l}1.36 \\
3.31\end{array}$ & $\begin{array}{l}1,940 \\
1,040\end{array}$ & $\begin{array}{r}6 \\
14\end{array}$ & $\begin{array}{l}108 \\
102\end{array}$ & $\begin{array}{l}21.4 \\
55.2\end{array}$ & 52 \\
\hline 11 & 1.90 & $\begin{array}{l}\text { Control } \\
\text { Dextran }\end{array}$ & $\begin{array}{l}116 / 60 \\
120 / 54\end{array}$ & $\begin{array}{l}80 \\
82\end{array}$ & $\begin{array}{l}6.20 \\
9.68\end{array}$ & $\begin{array}{l}3.26 \\
5.09\end{array}$ & $\begin{array}{r}1,030 \\
628\end{array}$ & $\begin{array}{l}0 \\
6\end{array}$ & $\begin{array}{l}96 \\
90\end{array}$ & $\begin{array}{r}64.6 \\
107.2\end{array}$ & \\
\hline 12 & 1.73 & $\begin{array}{l}\text { Control } \\
\text { Dextran }\end{array}$ & $\begin{array}{l}70 / 46 \\
86 / 50\end{array}$ & $\begin{array}{l}56 \\
64\end{array}$ & $\begin{array}{l}3.16 \\
5.00\end{array}$ & $\begin{array}{l}1.83 \\
2.89\end{array}$ & $\begin{array}{r}1,362 \\
945\end{array}$ & $\begin{array}{l}2 \\
5\end{array}$ & $\begin{array}{l}90 \\
86\end{array}$ & $\begin{array}{l}35.2 \\
58.1\end{array}$ & 70 \\
\hline 13 & 2.02 & $\begin{array}{l}\text { Control } \\
\text { Dextran }\end{array}$ & $\begin{array}{l}88 / 40 \\
88 / 44\end{array}$ & $\begin{array}{l}60 \\
54\end{array}$ & $\begin{array}{l}3.92 \\
4.78\end{array}$ & $\begin{array}{l}1.94 \\
2.36\end{array}$ & $\begin{array}{r}1,169 \\
788\end{array}$ & $\begin{array}{l}4 \\
8\end{array}$ & $\begin{array}{l}42 \\
48\end{array}$ & $\begin{array}{l}93.3 \\
99.5\end{array}$ & 59 \\
\hline 14 & 1.70 & $\begin{array}{l}\text { Control } \\
\text { Dextran }\end{array}$ & $\begin{array}{l}72 / 46 \\
98 / 66\end{array}$ & $\begin{array}{l}56 \\
78\end{array}$ & $\begin{array}{l}8.45 \\
8.89\end{array}$ & $\begin{array}{l}4.97 \\
5.23\end{array}$ & $\begin{array}{l}464 \\
628\end{array}$ & $\begin{array}{l}7 \\
8.4\end{array}$ & $\begin{array}{l}102 \\
102\end{array}$ & $\begin{array}{l}82.8 \\
87.2\end{array}$ & 125 \\
\hline 15 & 1.65 & $\begin{array}{l}\text { Control } \\
\text { Dextran }\end{array}$ & $\begin{array}{l}104 / 60 \\
144 / 80\end{array}$ & $\begin{array}{r}78 \\
108\end{array}$ & $\begin{array}{r}7.94 \\
12.82\end{array}$ & $\begin{array}{l}4.81 \\
7.77\end{array}$ & $\begin{array}{l}750 \\
612\end{array}$ & 10 & $\begin{array}{l}102 \\
108\end{array}$ & $\begin{array}{r}77.8 \\
189.0\end{array}$ & 73 \\
\hline 16 & 1.96 & $\begin{array}{l}\text { Control } \\
\text { Dextran }\end{array}$ & $\begin{array}{l}130 / 64 \\
148 / 70\end{array}$ & $\begin{array}{r}84 \\
100\end{array}$ & $\begin{array}{l}9.62 \\
8.81\end{array}$ & $\begin{array}{l}4.91 \\
4.49\end{array}$ & $\begin{array}{l}716 \\
790\end{array}$ & $\begin{array}{l}0 \\
7\end{array}$ & $\begin{array}{l}102 \\
100\end{array}$ & $\begin{array}{l}94.3 \\
88.1\end{array}$ & 54 \\
\hline 17 & 1.94 & $\begin{array}{l}\text { Control } \\
\text { Dextran }\end{array}$ & $\begin{array}{l}104 / 52 \\
104 / 56\end{array}$ & $\begin{array}{l}68 \\
60\end{array}$ & $\begin{array}{r}9.29 \\
10.00\end{array}$ & $\begin{array}{l}4.79 \\
5.15\end{array}$ & $\begin{array}{l}551 \\
400\end{array}$ & $\begin{array}{r}4 \\
10\end{array}$ & $\begin{array}{l}90 \\
78\end{array}$ & $\begin{array}{l}103.2 \\
128.2\end{array}$ & 57 \\
\hline 18 & 1.80 & $\begin{array}{l}\text { Control } \\
\text { Dextran }\end{array}$ & $\begin{array}{r}80 / 42 \\
108 / 56\end{array}$ & $\begin{array}{l}54 \\
70\end{array}$ & $\begin{array}{l}17.05 \\
10.64\end{array}$ & $\begin{array}{l}9.47 \\
5.91\end{array}$ & $\begin{array}{l}244 \\
450\end{array}$ & $\begin{array}{r}2 \\
10\end{array}$ & $\begin{array}{l}90 \\
90\end{array}$ & $\begin{array}{l}189.5 \\
118.2\end{array}$ & 72 \\
\hline 19 & 1.69 & $\begin{array}{l}\text { Control } \\
\text { Dextran }\end{array}$ & $\begin{array}{l}116 / 76 \\
136 / 84\end{array}$ & $\begin{array}{l}100 \\
110\end{array}$ & $\begin{array}{l}7.45 \\
8.73\end{array}$ & $\begin{array}{l}4.41 \\
5.16\end{array}$ & $\begin{array}{r}1,072 \\
854\end{array}$ & $\begin{array}{r}0 \\
17\end{array}$ & $\begin{array}{l}90 \\
90\end{array}$ & $\begin{array}{l}82.8 \\
97.0\end{array}$ & 62 \\
\hline 20 & 1.82 & $\begin{array}{l}\text { Control } \\
\text { Dextran }\end{array}$ & $\begin{array}{l}74 / 42 \\
86 / 50\end{array}$ & $\begin{array}{l}56 \\
62\end{array}$ & $\begin{array}{l}7.18 \\
8.88\end{array}$ & $\begin{array}{l}3.94 \\
4.88\end{array}$ & $\begin{array}{l}596 \\
450\end{array}$ & 13 & $\begin{array}{l}94 \\
92\end{array}$ & $\begin{array}{l}76.3 \\
96.5\end{array}$ & \\
\hline 21 & 1.91 & $\begin{array}{l}\text { Control } \\
\text { Dextran }\end{array}$ & $\begin{array}{r}74 / 44 \\
112 / 58\end{array}$ & $\begin{array}{l}57 \\
74\end{array}$ & $\begin{array}{l}12.06 \\
18.02\end{array}$ & $\begin{array}{l}6.31 \\
9.43\end{array}$ & $\begin{array}{l}362 \\
295\end{array}$ & $\begin{array}{l}2.5 \\
7.5\end{array}$ & $\begin{array}{l}82 \\
90\end{array}$ & $\begin{array}{l}147.0 \\
200.0\end{array}$ & 101 \\
\hline
\end{tabular}


TABLE III

Renal hemodynamics

\begin{tabular}{|c|c|c|c|c|c|c|c|}
\hline Patient & Period & $\begin{array}{c}\text { Mean arterial } \\
\text { pressure }\end{array}$ & $\begin{array}{l}\text { Renal blood } \\
\text { flow }\end{array}$ & $\begin{array}{c}\text { Renal } \\
\text { fraction }\end{array}$ & $\begin{array}{l}\text { Renal } \\
\text { resistance }\end{array}$ & $\begin{array}{c}\text { Renal venous } \\
\text { pressure }\end{array}$ & $\begin{array}{c}\text { Renal blood } \\
\text { volume }\end{array}$ \\
\hline & & $m m \mathrm{Hg}$ & $\underset{k i d n e y}{m l / m i n}$ per & $\%$ & dynes-sec-cm-s & $m m \mathrm{Hg}$ & $m l$ \\
\hline 1 & $\begin{array}{l}\text { Control } \\
\text { Dextran }\end{array}$ & $\begin{array}{l}84 \\
80\end{array}$ & $\begin{array}{l}372 \\
945\end{array}$ & $\begin{array}{l}20.7 \\
22.9\end{array}$ & $\begin{array}{l}7,100 \\
2,419\end{array}$ & $\begin{array}{l}18 \\
23\end{array}$ & \\
\hline 2 & $\begin{array}{l}\text { Control } \\
\text { Dextran }\end{array}$ & $\begin{array}{r}94 \\
104\end{array}$ & $\begin{array}{l}280 \\
595\end{array}$ & $\begin{array}{l}12.0 \\
15.9\end{array}$ & $\begin{array}{r}10,700 \\
5,850\end{array}$ & $\begin{array}{l}19 \\
17\end{array}$ & $\begin{array}{l}51.5 \\
83.8\end{array}$ \\
\hline 3 & $\begin{array}{l}\text { Control } \\
\text { Dextran }\end{array}$ & $\begin{array}{l}88 \\
94\end{array}$ & $\begin{array}{l}334 \\
588\end{array}$ & $\begin{array}{l}14.1 \\
14.6\end{array}$ & $\begin{array}{l}9,373 \\
5,310\end{array}$ & $16^{9.5}$ & $\begin{array}{l}38.2 \\
65.0\end{array}$ \\
\hline 4 & $\begin{array}{l}\text { Control } \\
\text { Dextran }\end{array}$ & $\begin{array}{l}62 \\
70\end{array}$ & $\begin{array}{l}219 \\
577\end{array}$ & $\begin{array}{r}9.9 \\
18.4\end{array}$ & $\begin{array}{l}9,140 \\
4,021\end{array}$ & $\begin{array}{l}12 \\
12\end{array}$ & $\begin{array}{l}30.0 \\
52.8\end{array}$ \\
\hline 5 & $\begin{array}{l}\text { Control } \\
\text { Dextran }\end{array}$ & $\begin{array}{l}56 \\
58\end{array}$ & $\begin{array}{l}220 \\
347\end{array}$ & $\begin{array}{l}6.8 \\
7.1\end{array}$ & $\begin{array}{l}8,360 \\
5,530\end{array}$ & $\begin{array}{l}10 \\
10\end{array}$ & $\begin{array}{l}53.7 \\
72.6\end{array}$ \\
\hline 6 & $\begin{array}{l}\text { Control } \\
\text { Dextran }\end{array}$ & $\begin{array}{l}66 \\
84\end{array}$ & $\begin{array}{l}171 \\
255\end{array}$ & $\begin{array}{l}6.2 \\
4.7\end{array}$ & $\begin{array}{l}12,180 \\
10,660\end{array}$ & $\begin{array}{l}14 \\
16\end{array}$ & $\begin{array}{l}39.5 \\
38.8\end{array}$ \\
\hline 7 & $\begin{array}{l}\text { Control } \\
\text { Dextran }\end{array}$ & $\begin{array}{l}52 \\
62\end{array}$ & $\begin{array}{r}85 \\
213\end{array}$ & $\begin{array}{l}4.8 \\
4.4\end{array}$ & $\begin{array}{l}21,600 \\
10,500\end{array}$ & $\begin{array}{l}6 \\
6\end{array}$ & $\begin{array}{l}52.6 \\
83.1\end{array}$ \\
\hline 8 & $\begin{array}{l}\text { Control } \\
\text { Dextran }\end{array}$ & $\begin{array}{l}96 \\
92\end{array}$ & $\begin{array}{l}129 \\
947\end{array}$ & $\begin{array}{r}4.6 \\
26.2\end{array}$ & $\begin{array}{r}28,000 \\
3,530\end{array}$ & $\begin{array}{l}5.5 \\
8.5\end{array}$ & $\begin{array}{r}33.4 \\
205.0\end{array}$ \\
\hline 15 & $\begin{array}{l}\text { Control } \\
\text { Dextran }\end{array}$ & $\begin{array}{r}78 \\
108\end{array}$ & $\begin{array}{l}243 \\
150\end{array}$ & $\begin{array}{l}6.1 \\
2.3\end{array}$ & $\begin{array}{l}10,880 \\
23,800\end{array}$ & $\begin{array}{l}12 \\
19\end{array}$ & $\begin{array}{l}37.3 \\
19.8\end{array}$ \\
\hline 16 & $\begin{array}{l}\text { Control } \\
\text { Dextran }\end{array}$ & $\begin{array}{r}84 \\
100\end{array}$ & $\begin{array}{l}298 \\
539\end{array}$ & $\begin{array}{r}6.2 \\
12.2\end{array}$ & $\begin{array}{l}9,120 \\
5,940\end{array}$ & $\begin{array}{l}16 \\
20\end{array}$ & $\begin{array}{l}37.5 \\
54.4\end{array}$ \\
\hline 17 & $\begin{array}{l}\text { Control } \\
\text { Dextran }\end{array}$ & $\begin{array}{l}68 \\
60\end{array}$ & $\begin{array}{l}434 \\
446\end{array}$ & $\begin{array}{l}9.4 \\
8.9\end{array}$ & $\begin{array}{l}5,520 \\
4,310\end{array}$ & $\begin{array}{r}8 \\
12\end{array}$ & $\begin{array}{l}53.1 \\
67.6\end{array}$ \\
\hline 18 & Control & 54 & 910 & 10.6 & 2,022 & 8 & 59.1 \\
\hline 19 & $\begin{array}{l}\text { Control } \\
\text { Dextran }\end{array}$ & $\begin{array}{l}100 \\
110\end{array}$ & $\begin{array}{l}177 \\
367\end{array}$ & $\begin{array}{l}4.8 \\
8.4\end{array}$ & $\begin{array}{l}20,340 \\
10,027\end{array}$ & $\begin{array}{l}10 \\
18\end{array}$ & $\begin{array}{l}46.2 \\
56.3\end{array}$ \\
\hline 20 & $\begin{array}{l}\text { Control } \\
\text { Dextran }\end{array}$ & $\begin{array}{l}56 \\
62\end{array}$ & $\begin{array}{l}238 \\
342\end{array}$ & $\begin{array}{l}6.3 \\
7.7\end{array}$ & $\begin{array}{l}5,972 \\
5,497\end{array}$ & $\begin{array}{l}13 \\
15\end{array}$ & $\begin{array}{l}33.2 \\
32.4\end{array}$ \\
\hline 21 & $\begin{array}{l}\text { Control } \\
\text { Dextran }\end{array}$ & $\begin{array}{l}58 \\
72\end{array}$ & $\begin{array}{l}1,028 \\
1,516\end{array}$ & $\begin{array}{l}16.0 \\
17.0\end{array}$ & $\begin{array}{l}1,907 \\
1,543\end{array}$ & $\begin{array}{c}9 \\
13.5\end{array}$ & $\begin{array}{l}126.0 \\
128.0\end{array}$ \\
\hline
\end{tabular}

that flow could not be accurately measured. In the other subjects the dye curves were usually of normal contour with exponential washouts. Control $\mathrm{RBF}$ in individual kidneys ranged from 85 to $1028 \mathrm{ml} / \mathrm{min}$ and averaged $343 \mathrm{ml} / \mathrm{min}$. RR averaged 10,937 dynes-sec-cm ${ }^{-5}, \mathrm{RF}$ was $9.1 \%$, and RBV averaged $49.4 \mathrm{ml} /$ kidney. RVP averaged $11.6 \mathrm{~mm} \mathrm{Hg}$ and was considerably higher than RAP. The elevation of RVP appeared to be related to the tenseness of the ascites.

A significant correlation was noted between $\mathrm{RR}$ and SVR $(r=0.630, P<0.01)$. Since SVR includes RR, a correlation between the two might be accidental. Nonrenal systemic vascular resistance
(NRVR) was therefore calculated using the formula :

$$
\mathrm{NRVR}=\frac{(\mathrm{MAP}-\mathrm{RAP}) \times 1332 \times 60}{\mathrm{CO}-(\mathrm{RBF} \times 2)} .
$$

RR was also significantly correlated with NRVR (Fig. 1).

RBF averaged $225 \mathrm{ml} /$ kidney per min in eight patients in Group I and $475 \mathrm{ml} / \mathrm{min}$ in seven patients in Group II. Two patients in Group II (Nos. 18 and 21) were oliguric with high RBF. These two patients had the highest control CO in the series. RBF was strikingly reduced in the other 13 patients. Flow was less than half the 


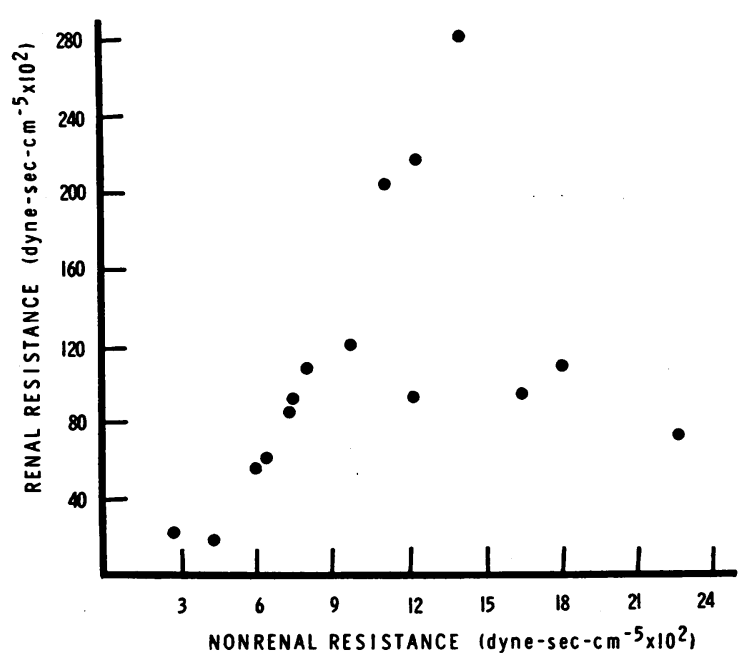

Fig. 1. RELATIONShip BETWEen RENAL VASCULAR RESISTANCE AND TOTAL SYSTEMIC NONRENAL RESISTANCE IN 15 PATIENTS. $r=0.625$.

predicted normal $(600 \mathrm{ml} / \mathrm{min}$ for individual kidneys) in six Group I patients and four Group II patients.

$\mathrm{RBF}$ was measured in seven hypotensive and eight normotensive patients. The two patients with high RBF were severely hypotensive. In the other five hypotensive subjects $\mathrm{RBF}$ was less than $250 \mathrm{ml} / \mathrm{min}$, averaging $187 \mathrm{ml} / \mathrm{min}$. RBF averaged $283 \mathrm{ml} / \mathrm{min}$ in the normotensive group.

Renal blood volume (RBV) ranged from 30 to $126 \mathrm{ml} /$ kidney and averaged $49.4 \mathrm{ml} /$ kidney, a value similar to previous figures for man (8). Renal blood volume in milliliters per $100 \mathrm{~g}$ of kidney weight was calculated in nine subjects in whom postmortem renal weights were obtained (Table IV). The calculated blood volume would overestimate intrarenal volume by the quantity of

TABLE IV

Renal blood volume

\begin{tabular}{cccc}
\hline \hline Patient & $\begin{array}{c}\text { Renal blood } \\
\text { volume }\end{array}$ & Kidney weight & $\begin{array}{c}\text { RBV/ } \\
\text { kidney wt }\end{array}$ \\
\hline & $m l$ & $g$ & $\mathrm{ml} / 100 \mathrm{~g}$ \\
2 & 45.5 & 160 & 28 \\
3 & 43.2 & 90 & 48 \\
5 & 51.9 & 255 & 21 \\
7 & 49.2 & 270 & 19 \\
16 & 33.1 & 110 & 30 \\
17 & 47.4 & 255 & 19 \\
18 & 56.8 & 240 & 24 \\
20 & 31.6 & 250 & 13 \\
21 & 114.0 & 200 & 57 \\
\hline
\end{tabular}

extrarenal blood contained in the renal artery and renal vein between injection and sampling sites. However, RBV in these nine kidneys averaged $30.2 \mathrm{ml} / 100 \mathrm{~g}$, a value only slightly higher than previous estimates in dogs (13).

\section{Dextran infusion}

A. Systemic effects. Immediately after infusion of $500 \mathrm{ml}$ of dextran 40, CO was increased in 19 of the 21 patients. CO rose an average of

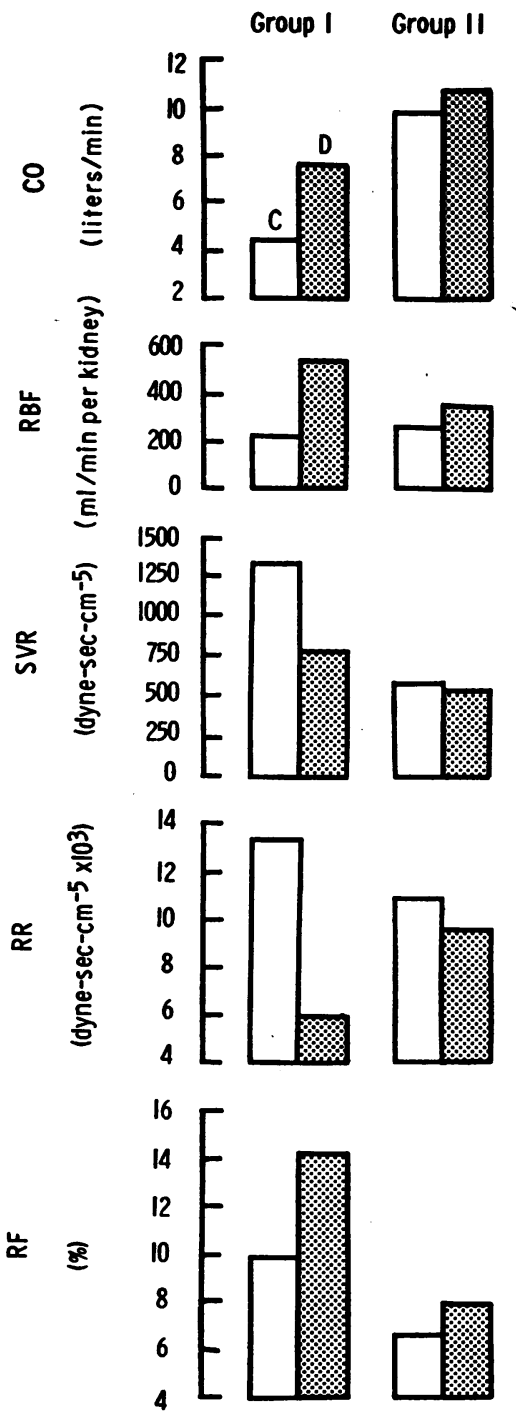

Fig. 2. EFFect OF DEXTRAN (D) ON SYSTEMIC AND RENAL HEMODYNAMICS. Group I, control (C) cardiac in$\operatorname{dex}(\mathrm{CI})$ less than 3.7 liters/min; Group II, CI greater than 3.9 liters/min. $\mathrm{CO}$, cardiac output; $\mathrm{RBF}$, renal blood flow per kidney; SVR, systemic vascular resistance; $R R$, renal resistance; $R F$, renal fraction. 


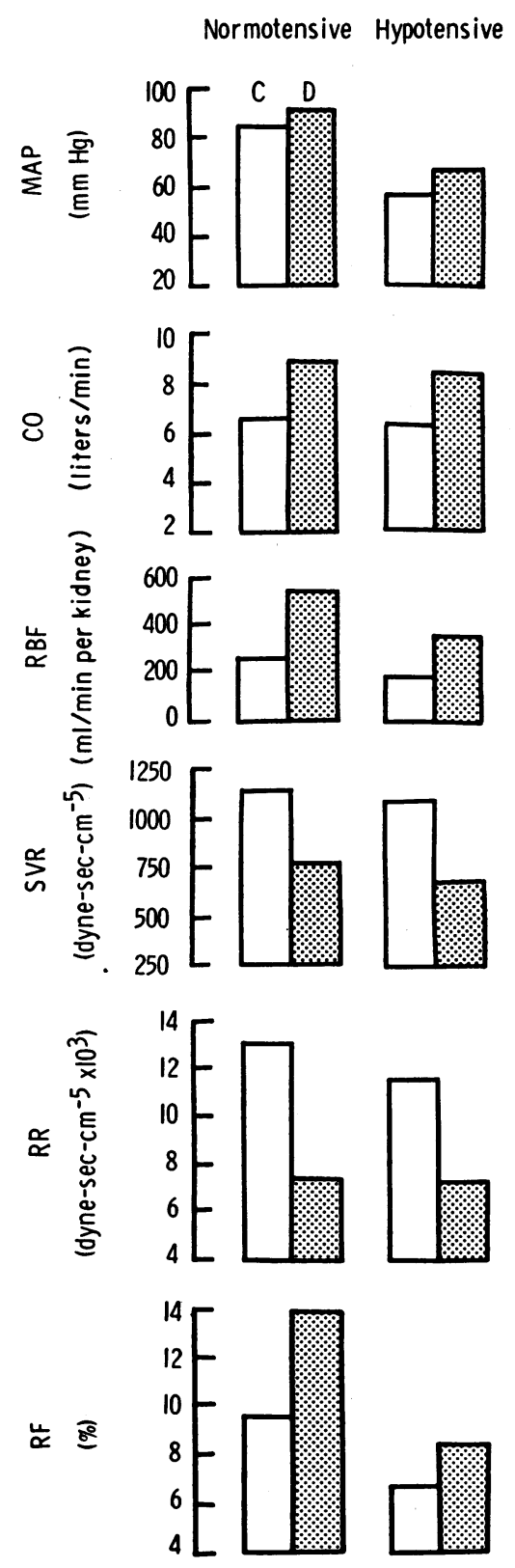

Fig. 3. Comparison of the efFect of DEXTRAN ON SYSTEMIC AND RENAL HEMODYNAMICS IN PATIENTS WITH SYSTOLIC ARTERIAL PRESSURE GREATER OR LESS THAN 100 MM HG. MAP, mean arterial pressure.

3.2 liters/min $(73 \%)$ in the 13 patients in Group $\mathrm{I}$ and only $0.9 \mathrm{liters} / \mathrm{min}(10 \%)$ in the eight patients in Group II (Fig. 2). SVR fell sharply in Group I, but was essentially unaltered in Group II. Dextran increased RAP from an average of 1.3 to $7.1 \mathrm{~mm} \mathrm{Hg}$ in Group I, whereas Group II patients started with a higher mean RAP of 2.8 $\mathrm{mm} \mathrm{Hg}$ and exhibited an increase to $10.4 \mathrm{~mm} \mathrm{Hg}$.

CO increased more than $40 \%$ after dextran infusion in all Group I patients except No. 13, whose CI remained low (2.36 liters/min) with an elevated RAP of $8 \mathrm{~mm} \mathrm{Hg}$. Intravenous administration of deslanoside, $0.6 \mathrm{mg}$, increased CI to 2.92 liters/min, suggesting that impaired myocardial performance may have been a factor in the poor response to volume expansion in this patient.

No difference was observed between the hypotensive and normotensive patients in their systemic hemodynamic response to dextran. MAP increased from an average of 85.5 to $92.0 \mathrm{~mm} \mathrm{Hg}$ in the nine normotensive subjects and from 56.8 to $67.7 \mathrm{~mm} \mathrm{Hg}$ in the hypotensive patients. $\mathrm{CO}$ and SVR changes were nearly identical (Fig. 3).

Volume expansion had a prominent effect on the pressure gradient from the inferior vena cava to the right atrium. High resistance to flow related to ascites and probable caval compression was manifested by a renal vein-right atrial pressure drop averaging $9.7 \mathrm{~mm} \mathrm{Hg}$ in the control period. The gradient was highest in patients with tense ascites. After dextran infusion, the pressure drop averaged only $5.7 \mathrm{~mm} \mathrm{Hg}$. Since $\mathrm{CO}$ and presumably caval flow were higher after dextran, the reduction in pressure gradient indicates a marked fall in resistance to flow in the inferior caval system.

The plasma volume-expanding effect of $500 \mathrm{ml}$ of low molecular weight dextran was measured in five of the patients. Plasma volume was increased immediately after infusion by an average of $939 \mathrm{ml}$ (range from 630 to $1231 \mathrm{ml}$ ). Hematocrit fell by an average of 6.1 volume per cent.

$B$. Renal effects. RBF increased after dextran infusion in 12 of 14 patients studied. In eight patients in Group I, RBF increased an average of $333 \mathrm{ml} / \mathrm{min}(148 \%)$ and was restored to over $500 \mathrm{ml} / \mathrm{kidney}$ per $\mathrm{min}$ in five of the subjects. These patients exhibited a striking reduction in $\mathrm{RR}$ and an increase in $\mathrm{RF}$ from an average of 9.9 to $14.3 \%$ (Figs. 2 and 4 ).

In contrast, the five Group II patients with reduced $\mathrm{RBF}$ responded to dextran with an increase in flow of only $91 \mathrm{ml} / \mathrm{min}$. Only one exhibited a rise in $\mathrm{RBF}$ to over $500 \mathrm{ml} / \mathrm{min}$. $R R$ remained high in these patients and the $R F$ was 


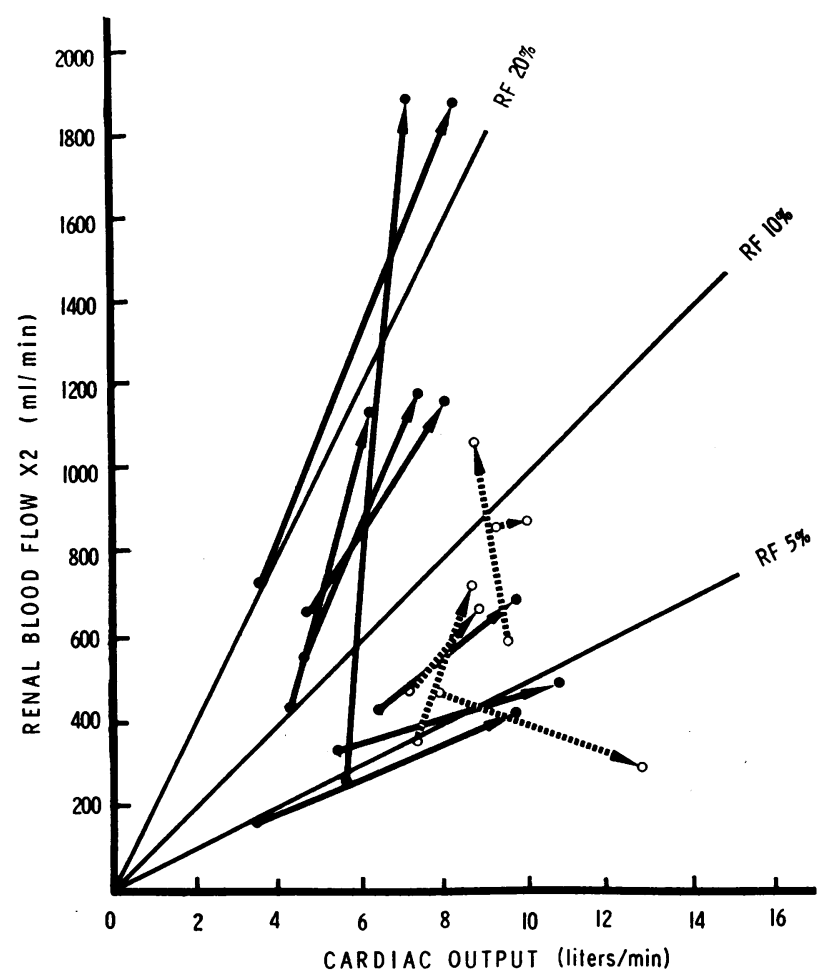

Fig. 4. RELATIONSHiP BETWEEN tOTAL RENAL BLOOD FLOW AND CARDIAC OUTPUT (RENAL FRACTION) AND THE DIRECTION OF CHANGE (ARROWS) AFTER VOLUME EXPANSION WITH DEXTRAN. Black dots and solid lines indicate patients in Group I. Open circles and interrupted lines indicate subjects in Group II. Group I patients show a greater increase in renal fraction after dextran.

not consistently altered (Figs. 2 and 4 ). Patient 21 had a high control $\mathrm{RBF}$ of $1028 \mathrm{ml} / \mathrm{min}$ that increased further after dextran to $1516 \mathrm{ml} / \mathrm{min}$. This increase in blood flow was associated with a sharp rise in urine output (see below and Fig. 5). The other patient with high RBF (No. 18) could not be restudied after volume expansion.

In normotensive subjects, $\mathrm{RBF}$ increased to an average of $572 \mathrm{ml} / \mathrm{min}$. In the hypotensive group, however, a flow of over $500 \mathrm{ml} / \mathrm{min}$ was restored in only one patient and the average $R B F$ in the five patients with low control $\mathrm{RBF}$ was increased to only $347 \mathrm{ml} / \mathrm{min}$ (Fig. 3).

RBV increased after dextran in 9 of the 13 subjects, rising by an average of $28.2 \mathrm{ml} /$ kidney.

\section{Ascites reinfusion}

In four patients ascites reinfusion was accomplished by pumping ascitic fluid by means of a
Sigmamotor pump from a polyethylene catheter in the peritoneal cavity to a closed bottle from which the fluid dripped by gravity through a blood filter into a catheter in the femoral vein. Reinfusion rate ranged from an estimated 300 to 1000 $\mathrm{ml} / \mathrm{hr}$. In two patients (Nos. 2 and 16), the procedure was performed immediately after the dextran infusion and both systemic and renal hemodynamics were monitored. In the other two patients (Nos. 3 and 11), the reinfusion was carried out at a later date and only systemic effects were studied.

In Patients 2, 3, and 11 (Group I), $\mathrm{CO}$ increased during the procedure $(2-20 \mathrm{hr}$ of reinfusion) from an average of 5.7 to 8.2 liters $/ \mathrm{min}$. $\mathrm{RBF}$ in Patient 2 was maintained during reinfusion at the same level $(574 \mathrm{ml} / \mathrm{min})$ that had previously been attained after dextran infusion (Fig. 6). In Patient 16 (Group II), CO fell 

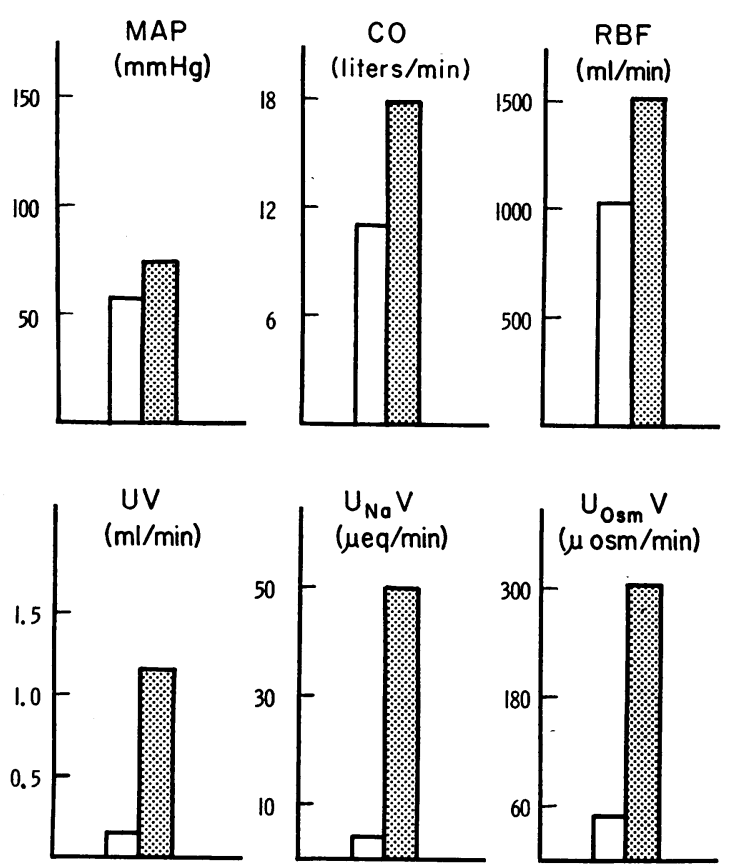

Fig. 5. EFFECT OF DEXTRAN ON HEMODYNAMICS AND URINARY EXCRETION RATES IN PATIENT 21. Despite high control RBF, dextran further increased systemic and renal hemodynamics and led to a sharp rise in sodium and osmolar excretion.

slightly during reinfusion as RAP increased precipitously, but $\mathrm{RBF}$ remained within normal range (Fig. 7) . The rapid rise in RAP and fall in $\mathrm{CO}$ suggest an element of myocardial failure during volume expansion. However, at autopsy the heart was of normal size without evidence of hypertrophy or dilatation.

Urine output increased promptly during ascites reinfusion in each of these oliguric patients. From control flow rates of $10-15 \mathrm{ml} / \mathrm{hr}$ urine output rose to $40-350 \mathrm{ml} / \mathrm{hr}$.

\section{Clinical observations}

Group I patients could not easily be differentiated from Group II patients on clinical grounds. The severity of oliguria was similar in the two groups. Blood urea nitrogen was slightly higher in Group I and total bilirubin was slightly higher in Group II, but these differences were not significant. Serum electrolyte and albumin levels were similar in the two groups, and ascites was of similar degree. Patients in Group II tended to present more obvious signs of high blood flow

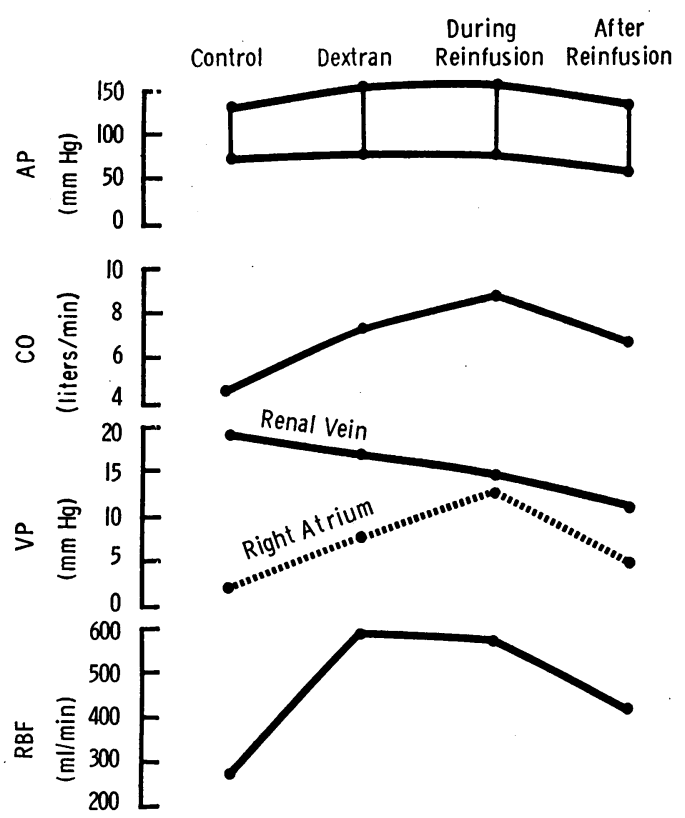

Fig. 6. EFfect of ascitic fluid Reinfusion in PATIENT 2. The increase in RBF during dextran infusion was sustained at nearly the same level during ascitic fluid reinfusion. The fall in $\mathrm{CO}$ and $\mathrm{RBF}$ after reinfusion indicates a transient hemodynamic effect. The decrease in hydraulic gradient between the right atrium and the renal vein after dextran and especially during reinfusion suggests reversal of caval collapse. AP, arterial pressure; VP, venous pressure.

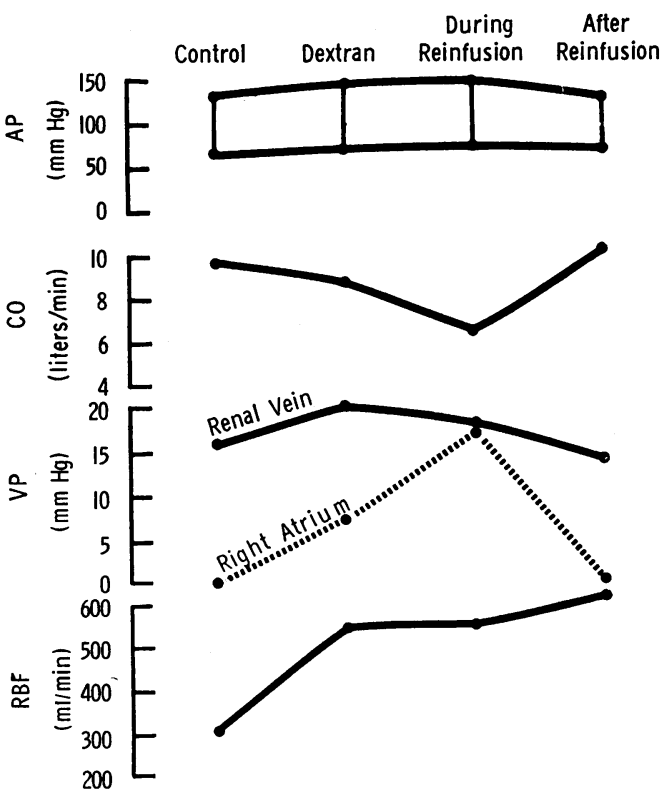

Fig. 7. Effect of ascitic fluid Reinfusion in PATIENT 16. Sustained hemodynamic improvement was present even after the reinfusion had stopped. 
and low peripheral resistance, including warm skin and bounding pulses. However, these signs were not invariably accurate in predicting the hemodynamic status of an individual patient.

13 of the 21 patients exhibited a significant increase in urine output following volume expansion. Urine flow increased in eight Group I patients and five Group II patients. The increase in urine output often was transient. Since an indwelling, bladder catheter was not routinely inserted in these patients, quantitative data for the group regarding urine and electrolyte excretion rates are not available. However, the response in Patient 21 (Fig. 5) is an example of a dramatic functional response to volume expansion with dextran. Although this patient had high control RBF he was markedly oliguric. After infusion of $500 \mathrm{ml}$ of dextran, urine flow and sodium excretion rose sharply.

In some patients oliguria persisted after dextran despite an improvement in renal hemodynamics. In no instance, however, did the infusion of dextran lead to a further reduction of urine output, as described recently in postsurgical patients given much larger amounts of low molecular weight dextran (14).

One patient (No. 19) made a complete recovery. The other 20 patients succumbed 13 days (average) after study (range from 1 to 54 days). Group I patients survived an average of 15.0 days. Group II patients had an average survival of 9.4 days. In hypotensive patients, survival averaged 10.2 days compared to the normotensive survival of 17.2 days $(P<0.1)$. While azotemia usually was present at the time of death, the major terminal event was more often gastrointestinal hemorrhage or hepatic coma.

\section{Discussion}

Previous investigators, using the $\mathrm{PAH}$ clearance method $(4,5,15,16)$, have found reduced RBF in patients with hepatic failure. However, clearance techniques may be inaccurate in the oliguric patient. Because PAH may be stored in the kidney at low urine flow rates, clearance measurements may significantly underestimate total $\mathrm{RBF}$ even when extraction ratios are determined (17). Tyler, Jeffries, and Wilder (18) reported low RBF using the nitrous oxide technique, but this method allows calculation of flow only in terms of kidney weight and does not lend itself to repeated measurements. Furthermore, nitrous oxide and other inert gas techniques measure only nutritive blood flow that perfuses capillary beds. The indicator-dilution method used in this study offers an accurate and almost instantaneous measurement of total RBF not dependent on urine production (8). Renal dye curves can be recorded in less than $20 \mathrm{sec}$ with removal of only $10 \mathrm{ml}$ of blood. Repeated measurements over a number of hours allow accurate assessment of changes in renal hemodynamics.

RBF was markedly reduced in 13 of 15 oliguric patients in this series, thus confirming previous conclusions that the renal failure of severe liver disease is usually accompanied by decreased renal perfusion. However, low total RBF may not be an invariable finding, since a high value for $R B F$ was measured in two patients in this series. Possible sources of error in the method must be considered before these high values for RBF can be accepted as valid. Misplacement of the renal arterial catheter to allow some dye to be injected into the aorta or of the renal venous catheter to allow contamination with vena caval blood would lead to falsely high values for RBF. However, smooth dye curves recorded in these patients suggest that the sampled blood was uniformly mixed, and close agreement between successive curves indicates that the same amount of dye entered the kidney after each injection. Overestimation of flow could also have resulted if significant quantities of injected indocyanine green became "trapped" in the kidney in a slowly circulating intravascular or extravascular space. Flow through inner medullary circuits might be increased in these patients, but it is unlikely that a significant portion of the injected dye would enter an intravascular space with a transit time longer than the recorded curves (19). A falsely high value for $R B F$ would also result if indocyanine green-labeled albumin equilibrated in a single renal circulation with an extravascular albumin compartment. Such a "loss" of intravascular albumin does not normally occur (20), but abnormally rapid extravascular distribution of albumin in these patients cannot be ruled out. Indeed, patient 21 had a somewhat larger calculated vascular volume than the other patients in 
this series, suggesting the possibility of an unusually large albumin space. The smooth renal curves and exponential indicator washout in the two patients with high $\mathrm{RBF}$, as well as in the other patients studied, make it unlikely that multiple renal arteries were the cause of an error in measured flow (8). Since the patients with high $\mathrm{RBF}$ had the highest values for $\mathrm{CO}$ in the series, it is likely that the RBF measurements were valid. Analysis of the significance of this observation of high $\mathrm{RBF}$ in this syndrome must await further confirmation.

While reduced renal perfusion may not be the only factor in the oliguria of severe liver disease, a number of investigators have considered this reduction of prime importance $(4,5)$. That renal vasoconstriction contributes to the low $\mathrm{RBF}$ in these patients was confirmed by the finding of high renal vascular resistance and a markedly reduced renal fraction of $\mathrm{CO}$. Previous investigators have discounted the importance of total blood flow as a factor in the renal hypoperfusion, since they have found $\mathrm{CO}$ normal or high in most of these patients (5). However, a high $\mathrm{CO}$ is usually observed in the hyperdynamic circulatory state of Laennec's cirrhosis (21), and even a normal $\mathrm{CO}$ in such a patient may represent a serious deficiency in nutritive blood flow. Elevations of the lactate-pyruvate ratios, indicative of tissue hypoxia, frequently have been found in patients with high $\mathrm{CO}$ and hepatic failure in this laboratory. ${ }^{2}$

The observation in this series of a significant correlation between renal resistance and nonrenal systemic vascular resistance suggests that the renal vasoconstriction is not an isolated vascular phenomenon but rather a manifestation of a more generalized vasoconstriction that results from a relatively reduced $\mathrm{CO}$. Furthermore, since $\mathrm{CO}$ was correlated with blood volume, it might be hypothesized that renal vasoconstriction could be related to a reduction in $\mathrm{CO}$ and a rise in systemic vascular resistance that results from reduced circulating volume (22). This pathophysiological mechanism appeared to be playing an important role in some of the subjects studied. After volume expansion systemic vascular resistance fell in 18 of 21 patients and renal resistance was

2 Cohn, J. N, Unpublished observations, reduced in 13 of 14 . The response was most dramatic in patients in Group I, who had lower control cardiac outputs and blood volumes. Dextran infusion increased $\mathrm{CO}$ in these subjects by an average of $73 \%$ and in many patients it restored $R B F$ to a normal range. Since $R R$ fell more sharply than systemic resistance, the renal fraction rose. The response in these patients suggests that volume depletion was a significant factor in their circulatory abnormality.

The reduced circulating blood volume in these patients might be attributed to a number of factors, including hypoalbuminemia, fever, infection, diuretic therapy, rapid accumulation of ascitic fluid, pooling in dilated hepatic lymphatics, capillary transudation in the lower body because of increased venous pressure resulting from ascites, and unrecognized gastrointestinal hemorrhage. The removal of small amounts of ascitic fluid may have been a possible factor in two of these patients. Whatever the cause of the hypovolemia in these patients, it is apparently difficult to maintain a blood volume adequate for optimum circulation. The hemodynamic and clinical response to dextran infusion was often transient, and whole blood or albumin was often used when hypovolemia recurred. In four patients, ascitic fluid was reinfused in an attempt to restore volume, and perhaps to also correct a possible hemodynamic cause for recurrent hypovolemia. While this treatment has occasionally produced sustained clinical, as well as hemodynamic improvement in our hands (23) (Fig. 7), the circulatory response may be tratisient as shown by the tendency for $\mathrm{CO}$ and $\mathrm{RBF}$ to fall toward control levels after the reinfusion was stopped in Patient 2 (Fig. 6).

In the Group II patients, volume depletion apparently was not an important factor in their oliguric state, since they were normovolemic and the high CI did not increase further after dextran. Whereas RBF was high in two of the patients, a moderate to severe reduction in others was not corrected by volume expansion. Thus, a mechanism other than volume depletion may contribute to renal vasoconstriction in some patients with liver failure. Neurogenic (24) and humoral (25) factors have been implicated in the genesis of the renal hypoperfusion. Irrespective of cause, however, preliminary studies in this laboratory have 
indicated that the renal vasoconstriction can be reversed with renal arterial infusion of vasodilator agents (26).

Since the control RBV was not significantly different from normal RBV, either in total volume or relative to kidney weight, the abnormalities in intrarenal hemodynamics apparently were not manifested by gross alterations in intravascular volume. After dextran infusion, there was a significant increase in RBV. The hyperoncotic nature of the $10 \%$ dextran, especially in the presence of hypoalbuminemia, would enhance the movement of fluid from the renal interstitium into the intravascular compartment, thus reducing renal tissue pressure and increasing the capacity of the vascular bed.

Increased plasma oncotic pressure during infusion of the $10 \%$ dextran resulted in mobilization of extravascular fluid and expansion of the plasma volume by an amount considerably greater than the $500 \mathrm{ml}$ administered. In addition to the volume effects of dextran, rheological properties of the lower molecular weight particles have been reported to exert a beneficial effect on microcirculatory flow in some situations (27). Dextran not only inhibits red cell sludging (28) but also lowers whole blood viscosity both by its direct effect and by lowering the hematocrit (29). Whereas these properties of dextran may have contributed to the hemodynamic responses observed, most of the effects in these patients probably can be attributed to expansion of the circulating volume.

The relationship between the hemodynamic abnormalities demonstrated in these patients and their renal failure must remain speculative. Renal vasoconstriction and systemic hypotension may lead to enhanced tubular reabsorption of filtrate, and the reduction of urine flow could contribute to tubular damage $(30,31)$. The failure of urine output always to increase, despite restoration of apparently normal total renal blood flow, could be explained either by the transient nature of the circulatory response to volume expansion or by the possibility that functional tubular damage had already developed in these patients. However, the possible influence of alterations in intrarenal distribution of blood flow and of changes in glomerular function must also be considered.
Although these studies may contribute to an understanding of renal hemodynamics in this syndrome, they offer little encouragement in the clinical management of these patients. Improvement in the renal blood flow by volume expansion or direct renal vasodilatation (26) and correction of systemic hypotension with the administration of vasoconstrictor agents $(32,33)$ may restore urine output. Since these patients usually succumb before frank uremia develops, however, correction of the oliguria will probably be of no lasting benefit unless hepatic function also improves.

\section{Acknowledgment}

We gratefully acknowledge the technical assistance of Mrs. Barbara Leibel, Mrs. Ann Crossin, and Mr. Aldo Notargiacomo.

\section{References}

1. Summerskill, W. H. J. 1966. Hepatic failure and the kidney. Gastroenterology. 51: 94.

2. Papper, S. 1958. The role of the kidney in Laennec's cirrhosis of the liver. Medicine. 37: 299.

3. Papper, S., J. L. Belsky, and K. H. Bleifer. 1959. Renal failure in Laennec's cirrhosis of the liver. I. Description of clinical and laboratory features. Ann. Internal Med. 51 : 759.

4. Baldus, W. P., W. H. J. Summerskill, J. C. Hunt, and and F. T. Maher. 1964. Renal circulation in cirrhosis: Observations based on catheterization of the renal vein. J. Clin. Invest. 43: 1090.

5. Lancestremere, R. G., P. L. Davidson, L. E. Earley, F. J. O'Brien, and S. Papper. 1962. Renal failure in Laennec's cirrhosis. II. Simultaneous determination of cardiac output and renal hemodynamics. J. Clin. Invest. 41: 1922.

6. Selkurt, E. E. 1962. Renal blood flow and renal clearances during hemorrhage and hemorrhagic shock. In Shock, Pathogenesis and Therapy. Springer-Verlag, Berlin. 145.

7. Vesin, P. 1962. Late functional renal failure in cirrhosis with ascites: pathophysiology, diagnosis and treatment. In Aktuelle Probleme der Hepatologie. G. A. Martini and S. Sherlock, editors. George Thieme Verlag, Stuttgart. 98.

8. Cohn, J. N., and E. A. Gombos. 1965. Unilateral renal hemodynamics studied by an indicator-dilution technic in man. Am. J. Cardiol. 16: 820.

9. Hamilton, W. F., J. W. Moore, J. M. Kinsman, and R. G. Spurling. 1932. Studies on the circulation. IV. Further analysis of the injection method and of changes in hemodynamics under physiological and pathological conditions. Am. J. Physiol. 99: 534. 
10. Owen, C. A., Jr., and M. H. Power. 1953. Intercellular plasma of centrifuged human erythrocytes as measured by means of iodo'albumin. J. Appl. Physiol. 5 : 323.

11. Gregersen, M. I., and R. A. Rawson. 1959. Blood volume. Physiol. Rev. 39 : 307.

12. Mainland, D. 1963. Elementary Medical Statistics. W. B. Saunders Co., Philadelphia. 2nd edition.

13. Selkurt, E. E. 1963. The renal circulation. In Handbook of Physiology. W. F. Hamilton, editor. American Physiological Society, Washington, D. C. 2: 1471.

14. Niall, J. F., and J. C. Doyle. 1966. Renal failure associated with dextran infusions. Proceedings 3rd International Congress of Nephrology, Washington, D. C., 25-30 September 1966. 248.

15. Onen, K. H. 1960. Renal hemodynamics in hepatic cirrhosis. Lancet. 1: 203.

16. Shear, L., P. W. Hall, III, and G. J. Gabuzda. 1965. Renal failure in patients with cirrhosis of the liver. II. Factors influencing maximal urinary flow rate. Am. J. Med. 39 : 199.

17. Balint, P., A. Fekete, and J. Forgacs. 1964. Quantitative considerations on the storage of clearance substances in the kidney. Clin. Sci. 26: 345 .

18. Tyler, J. M., J. L. Jeffries, and C. E. Wilder. 1962. A study of the renal blood flow by nitrous oxide technique in normal and oliguric patients with cirrhosis of liver. Clin. Res. 10: 194. (Abstr.)

19. Thurau, K. 1964. Renal hemodynamics. Am. J. Med. 36: 698.

20. Lilienfield, L. S., J. C. Rose, and N. A. Lassen. 1958. Diverse distribution of red cells and albumin in the dog kidney. Circulation Res. $6: 810$.

21. Kowalski, H. J., and W. H. Abelmann. 1953. The cardiac output at rest in Laennec's cirrhosis. $J$. Clin. Invest. 32 : 1025.

22. Cohn, J. N., and M. H. Luria. 1966. Studies in clinical shock and hypotension. IV. Variations in reflex vasoconstriction and cardiac stimulation. Circulation. $34: 823$.

23. Cohn, J. N. 1964. Hepatorenal failure following portocaval shunt: Hemodynamic considerations and the application of ascitic fluid infusion. Med. Ann. District Columbia. 33: 567.
24. Onnis, M., H. B. Shumacker, Jr., and G. Bounous. 1963. Blood pressure and renal blood flow response to occlusion of visceral arteries. Ann. Sur. 157 : 56.

25. Shorr, E., B. W. Zweifach, and R. F. Furchgott. 1948. Hepato-renal factors in circulatory homeostasis; influence of humoral factors of hepato-renal origin on vascular reactions to hemorrhage. Ann. N. Y. Acad. Sci. 49 : 571.

26. Tristani, F. E., and J. N. Cohn. 1966. Mechanism of renal vasoconstriction in hepatic failure. Clin. Res. 14 : 389. (Abstr.)

27. Gelin, L. E., and B. Ingelman. 1961. Rheomacrodex -a new dextran solution for rheological treatment of impaired capillary flow. Acta Chir. Scand. 122 : 294.

28. Bernstein, E. F., F. G. Emmings, R. L. Evans, A. Castaneda, and R. L. Varco. 1963. Effect of low molecular weight dextran on red blood cell charge during clinical extracorporeal circulation. Circulation. 27: 816.

29. Seaman, G. V. F., W. Hissen, L. Lino, and R. L. Swank. 1965. Physicochemical changes in blood arising from dextran infusions. Clin. Sci. 29: 293.

30. Teschan, P. E., and N. L. Lawson. 1966. Studies in acute renal failure. Prevention by osmotic diuresis and observations on the effect of plasma and extracellular volume expansion. Nephron. 3: 1.

31. Shear, L., J. Kleinerman, and G. J. Gabuzda. 1965. Renal failure in patients with cirrhosis of the liver. I. Clinical and pathologic characteristics. Am. J. Med. 39 : 184.

32. Lancestremere, R. G., E. L. Klinger, Jr., E. Frisch, and S. Papper. 1963. Simultaneous determination of cardiac output and renal function in patients with Laennec's cirrhosis during the administration of the pressor amine, metaraminol. J. Lab. Clin. Med. $61: 820$.

33. Cohn, J. N., F. E. Tristani, I. Khatri, and B. Leibel. 1967. Extra-renal vasoconstriction: A unique hemodynamic effect of PLV2 in the treatment of shock and sodium retention. Clin. Res. 15: 54. (Abstr.) 